\title{
Once Weekly Application of Urea $40 \%$ and Bifonazole $1 \%$ Leads to Earlier Nail Removal in Onychomycosis
}

\author{
Avner Shemer $^{a, b}$ Yossi Eshela, b Aditya K. Guptac, d Renata Farhi ${ }^{\mathrm{e}}$ \\ Eckart Haneke $^{f-i}$ Carlton Ralph Daniel ${ }^{j, k}$ Kelly Foley ${ }^{d}$ Emma M. Quinlan ${ }^{d}$

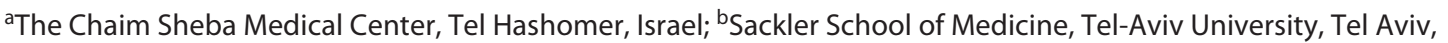 \\ Israel; ' CDepartment of Dermatology, Faculty of Medicine, University of Toronto, Toronto, ON, Canada; ${ }^{\mathrm{d}}$ Mediprobe

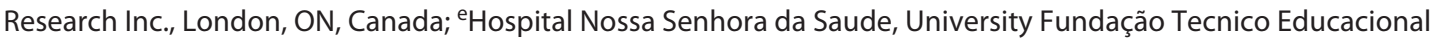 \\ Souza Marques, Rio de Janeiro, Brazil; 'Department of Dermatology, Inselspital, University of Bern, Bern, Switzerland; \\ gPrivate Dermatology Practice Dermaticum, Freiburg, Germany; ${ }^{\text {h} C e n t r o ~ d e ~ D e r m a t o l o g i ́ a ~ E p i d e r m i s, ~ I n s t i t u t o ~}$ \\ CUF, Porto, Portugal; 'Department of Dermatology, Ghent University Hospital, Ghent, Belgium; iDepartment of \\ Dermatology, University of Mississippi School of Medicine, Jackson, MS, USA; ${ }^{k}$ Department of Dermatology, School \\ of Medicine, University of Alabama, Birmingham, AL, USA
}

\section{Keywords}

Urea Bifonazole $\cdot$ Chemical avulsion - Onychomycosis .

Keratoplastic agent · Nail sealing · Nail treatment · AGISPOR ONYCHOSET

\begin{abstract}
Introduction: Onychomycosis is a chronic nail fungal infection resulting in nail damage and a decreased quality of life. Chemical avulsion of the nail with urea and bifonazole removes fungally infected debris, increasing antifungal treatment efficacy and penetration. Previous clinical observations describe patients who applied their urea and bifonazole ointment less frequently, achieving earlier nail removal. In this study, we analyzed the relationship between duration of urea and bifonazole application and time to nail avulsion. Methods: $x^{2}$ tests, multiple regression analysis, and ANOVA were performed to analyze the similarities between treatment regimens (daily, every 3 days, or once a week), association of regimens or patient characteristics to nail removal, and compare time to nail removal between each reg-
\end{abstract}

imen, respectively. Results: Daily application of ointment and sealing resulted in an average length of time $( \pm S D)$ to nail removal of 18.7 days ( \pm 6.8 days); once every 3 days resulted in nail removal at 12.7 days ( \pm 6.2 days) and once per week at 11 days ( \pm 4.46 days) ( $p<0.001)$. Age was the only patient factor that affected duration to nail removal. Conclusion: Once weekly application of ointment with sealing for a 1-week duration is associated with a decrease in time to complete chemical avulsion of the nail by approximately 1 week.

๑) 2020 S. Karger AG, Basel

\section{Introduction}

Onychomycosis is the most common nail disease in the world, constituting $50 \%$ of all nail infections [1]. It is a chronic fungal infection of the nail, often presenting as

Avner Shemer and Yossi Eshel contributed equally to the article.

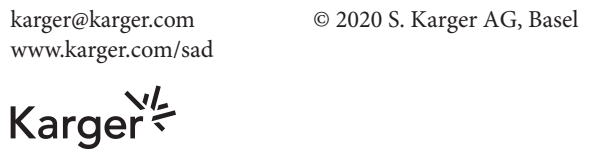

Aditya K. Gupta

Mediprobe Research Inc.

645 Windermere Road

London, ON N5X 2P1 (Canada)

agupta@execulink.com 
Table 1. Patient characteristics at baseline

\begin{tabular}{|c|c|c|c|c|}
\hline & \multicolumn{3}{|l|}{ Regimen } & \multirow[t]{2}{*}{$p$ value } \\
\hline & daily & every 3 days & weekly & \\
\hline Sample size at baseline & 55 & 58 & 64 & \\
\hline \multicolumn{5}{|l|}{ Gender, $n(\%)$} \\
\hline Male & $37(67.3)$ & $36(62.1)$ & $42(65.6)$ & 0.837 \\
\hline Female & $18(32.7)$ & $22(37.9)$ & $22(34.4)$ & \\
\hline Diabetes, $n(\%)$ & $8(14.5)$ & $6(10.3)$ & $8(12.5)$ & 0.729 \\
\hline \multicolumn{5}{|l|}{ Age, years, $n(\%)$} \\
\hline$<20$ & $3(5.5)$ & $1(1.7)$ & 0 & 0.121 \\
\hline $20-39$ & $25(45.5)$ & $28(48.3)$ & $22(34.4)$ & \\
\hline $40-59$ & $23(41.8)$ & $28(48.3)$ & $36(56.3)$ & \\
\hline $60+$ & $4(7.3)$ & $1(1.7)$ & $6(9.4)$ & \\
\hline \multicolumn{5}{|l|}{ Causative organism, $n(\%)$} \\
\hline Negative culture & $8(14.5)$ & $1(1.7)$ & $4(6.3)$ & 0.116 \\
\hline Dermatophyte & $33(60.0)$ & $43(74.1)$ & $44(68.8)$ & \\
\hline Nondermatophyte & $2(3.6)$ & $6(10.3)$ & $9(14.1)$ & \\
\hline Candida spp. & $2(3.6)$ & $2(3.4)$ & $1(1.6)$ & \\
\hline Mixed infection & $4(7.3)$ & $3(5.2)$ & $2(3.1)$ & \\
\hline Number of subjects who completed the study & 50 & 52 & 61 & \\
\hline
\end{tabular}

nail discoloration, nail plate hardening, and crumbling and hyperkeratosis of the nail bed, resulting in decreased quality of life for people with the condition $[2,3]$. The most common treatment for the disease is systemic antifungal drugs with a $24-69 \%$ failure rate, with certain treatments having hepatotoxic side effects and drug interactions $[4,5]$. Topical antifungals are therefore sometimes preferred due to localized activity; however, they must be applied for a minimum of 12 months for toenails, due to slow growth of nails and limited penetration of the drug through the nail [6].

The nail plate is predominantly composed of $\alpha$-keratin proteins which are organized into a structure mainly stabilized by hydrophobic connections, hydrogen bonds, and di-sulfide bonds [7]. While onychomycotic nails are less dense and more porous than healthy nails, the hydrophobic connections remain, preventing most topical onychomycosis therapies comprised of large, hydrophobic molecules, from penetrating to the sites of fungal infection [8]. Therefore, chemical removal or avulsion of a maximum amount of infected nails prior to antifungal treatment allows for higher healing rates in both systemic and topical treatments, due to reduced fungal load and increased penetration through the nail bed and plate $[9,10]$.

Urea is a keratolytic agent which is used in the chemical avulsion of fungally infected nails. The compound damages the structure of the proteins in the nail by decreasing the hydrophobic effect (due to displacing of wa- ter in the solvation shell and specifically binding to amide units), compromising the hydrogen bonds and softening the nail by hydration, thus reducing its tensile strength and enabling its mechanical removal [11-13]. AGISPOR ONYCHOSET is a marketed ointment containing urea $40 \%$ and the antifungal bifonazole $1 \%$. According to the ointment's standard treatment protocol, the patient is required to soak the nail in warm water for $10 \mathrm{~min}$, trim and scrape fungally infected portions of the nail, apply the ointment, and seal it with a bandage. The following day, the bandage is removed and the nail is soaked again, followed by scraping of fungal debris and reapplication of the ointment and bandage. This process is repeated daily [14]. In a previous randomized, double-blind comparison study with topical bifonazole-urea ointment monotherapy and in combination with oral griseofulvin, we noticed that patients who performed these actions less frequently achieved earlier removal of the nail [15]. Similar results were seen in our clinic, which led us to believe that better sealing leads to earlier chemical avulsion.

Therefore, we decided to test our hypothesis that a lower frequency of treatment administration and longer application time of 3 days or 1 week with a new sealing technique would lead to better and quicker results in the treatment of onychomycosis. We treated patients with a combination therapy that included maximal sealing of the nail using a new sealing technique with lower frequency of treatment administration. 
Table 2. Summary of multiple regression analysis $(N=160)$

\begin{tabular}{lrll}
\hline Variable & \multicolumn{1}{l}{$B$} & SE & $\beta$ \\
\hline Constant & 14.092 & 1.869 & \\
Age & 0.128 & 0.041 & $0.220^{\mathrm{a}}$ \\
Gender & -0.441 & 0.965 & -0.032 \\
Diabetes & -0.808 & 1.380 & -0.041 \\
Treatment regimen & -7.393 & 1.007 & $-0.504^{\mathrm{b}}$ \\
\hline
\end{tabular}

Gender is for males compared to females. Treatment regimen is for alternate regimens (every 3 days and weekly) compared to daily. $B$, unstandardized regression coefficient; SE, standard error of the coefficient; $\beta$, standardized coefficient. ${ }^{\mathrm{a}} p<0.01 .{ }^{\mathrm{b}} p<0.001$.

\section{Methods}

We conducted a retrospective cohort study on patients (18 to $>60$ years of age) visiting the "LEY HAYASMIN" clinic in Netanya, Israel, during the years 2009-2018. We screened 15,000 patients suspected of having moderate to severe toenail onychomycosis (25 to $\leq 75 \%$ of target nail involvement with thick, coarse toenails). These patients were subjected to a medical interview and a clinical evaluation of the infected nail(s), followed by laboratory confirmation with $\mathrm{KOH}$ microscopy and/or fungal culture. Exclusion criteria included patients sensitive to urea/bifonazole agents or the plaster, and pregnant women. Of all included patients diagnosed with onychomycosis $(n=177)$, the most common etiological agents were dermatophytes $(120,67.8 \%)$, nondermatophyte molds (17, 9.6\%), and Candida spp. (5, 2.8\%). Mixed infections were observed in $9(5.1 \%)$ patients. Nail samples taken from $13(7.3 \%)$ patients were $\mathrm{KOH}$ microscopy positive, but culture was negative. However, they were deemed as confirmed cases of onychomycosis and considered for treatment with the AGISPOR ONYCHOSET ointment. Of the 177 patients with onychomycosis, 115 were male $(65.0 \%)$ and 62 were female (35.0\%). Twenty-two of $177(12.4 \%)$ had a coexisting condition such as diabetes. Patient characteristics at baseline are summarized in Table 1.

Treatment was applied to trimmed nails after soaking in warm water for $10 \mathrm{~min}$ and scraping of the distal portion with a scraper provided along with the ointment kit. This study had 3 treatment groups which were created based on patients' reported ability to adhere to the specific treatment protocol. Group 1 had 55 patients, of whom 5 dropped out (thus, 33 males and 17 females completed the study); group 1 subjects were instructed to apply the urea $40 \%$ and bifonazole $1 \%$ ointment once a day and wrap the nail with a plaster provided along with the ointment kit, leaving the plaster on until the next application. Group 2 had 58 patients, of whom 6 dropped out (thus, 32 males and 20 females completed the study), and group 3 had 64 patients, of whom 3 dropped out (thus, 40 males and 21 females completed the study) who were instructed to apply the urea $40 \%$ and bifonazole $1 \%$ ointment once every 3 days and once a week, respectively, again leaving the plaster on until the next application. Both groups 2 and 3 had to wrap the nail following ointment application with a new sealing technique proposed by the dermatologist, that is, covering the nail with the plaster provided along with the ointment kit followed by wrapping another

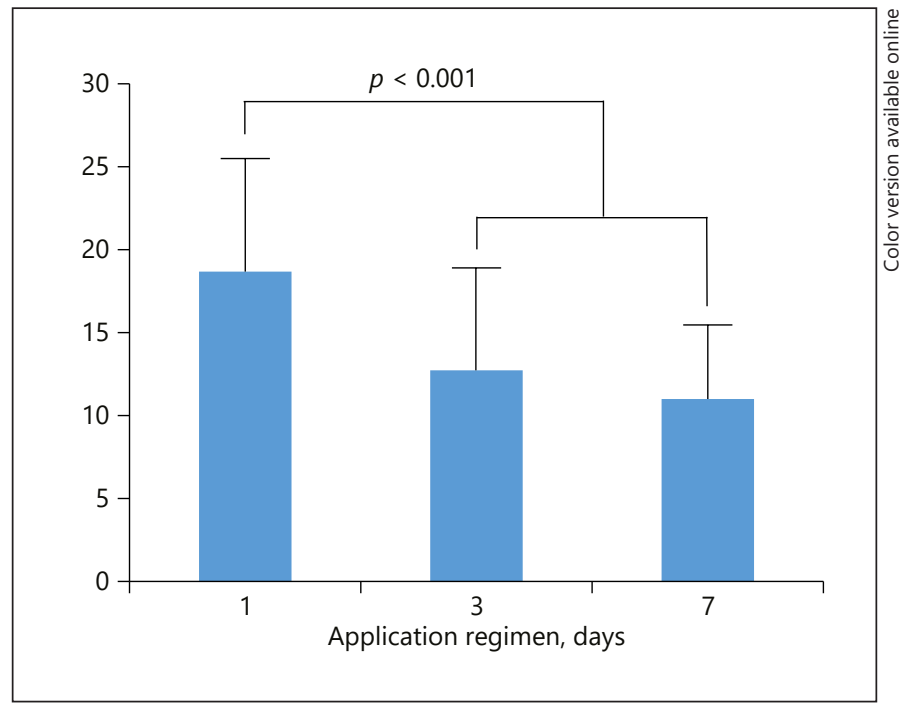

Fig. 1. Time to chemical avulsion. ${ }^{* *} p<0.001$.

layer of paper leukoplast tape around the toe which seals the middle of the toe and the nail, leaving the distal skin of the toe bare. Strict adhesion to the treatment protocol was expected of the patients until there were no more soft infected parts of the nail left to be trimmed and scraped: this process is known as chemical avulsion. The duration to complete avulsion of all infected portions of the nail in each group, patient compliance to the treatment, and any discomfort or side effects were asked to be reported to the consulting dermatologist.

A one-way ANOVA was used to compare the time to reach chemical avulsion with each of the 3 treatment regimens. $\chi^{2}$ tests were used to determine if there were differences between the treatment regimens for patient characteristics. A multiple regression was performed to determine whether treatment regimen, age, gender, and presence of diabetes predicted time to chemical avulsion. Analyses were performed using SPSS Statistics 20 (IBM) with significance set to $\alpha=0.05$.

\section{Results}

There were no differences between treatment groups in patient characteristics (Tables 1,2 ) and compliance to the treatment. Analyses were conducted on subjects who completed the study (i.e., drop outs were excluded from analyses). The average time to complete avulsion $( \pm \mathrm{SD})$ in group 1 with daily application of the urea $40 \%$ and bifonazole $1 \%$ ointment was 18.68 days ( \pm 6.81 days), group 2 with application once every 3 days was $12.73( \pm 6.17$ days) days, and group 3 with application once a week was 11 days $( \pm 4.46$ days $)(p<0.001)$. Figure 1 shows the time in days that was required for chemical avulsion for each treatment regimen. A one-way ANOVA was performed 
to determine if there were significant differences between treatment regimens in the time to reach chemical avulsion. There was a significant main effect of treatment regimen $(F(2,160)=25.51, p<0.001)$, with the alternative regimens of ointment application and sealing every 3 days or weekly producing chemical avulsion in significantly less time than the standard of daily ointment application and sealing (Tukey post hoc $p<0.001$ ). There was no significant difference between treatment every 3 days and weekly $(p=0.259)$.

A multiple regression was performed using the independent variables of age, gender, treatment regimen, and presence of diabetes. For treatment regimen, the alternate regimens of every 3 days and weekly were collapsed and compared to the standard daily treatment regimen. The regression model was significant $\left(F(4,155)=15.06, p<0.001\right.$, adjusted $\left.R^{2}=0.261\right)$. Gender and presence of diabetes did not significantly influence the time to chemical avulsion (Table 2). An increase in age of 1 year is associated with increasing the time to chemical avulsion by 0.128 days $(p<0.01)$. Treatment regimen significantly predicted time to chemical avulsion. Compared to daily application of ointment and sealing, alternate applications (every 3 days and weekly) of ointment and sealing are associated with decreasing the time to chemical avulsion by $\sim 7.4$ days ( $p<0.001$, Table 2$)$. Mild dermal irritation was reported by 2 patients in group 3 who applied the ointment once every 7 days, which did not prevent them from completing the treatment.

\section{Discussion}

The results of this study support our hypothesis that increased duration of urea $40 \%$ and bifonazole $1 \%$ ointment application before removing the plaster and reapplying the product leads to decreased overall treatment time until chemical avulsion of the mycotic nail. While reapplying the treatment daily resulted in an average of 18.68 days before chemical avulsion, this duration decreased to 12.73 days for a reapplication of every 3 days and decreased further to an average of 11 days for weekly reapplication. It appears that the efficacy of this treatment method begins to plateau between 3 and 7 days.

We hypothesized that the increased duration between applications and the tighter sealing method lead to a greater efficacy of chemical avulsion due to 2 main reasons. The first reason is due to the increased time of contact between the urea preparation and the nail plate, the urea solution has more time to penetrate into the more porous mycotic areas of the plate, resulting in higher keratolytic activity. When the solution is removed every day, the amount of solution absorbed is minimal; fresh application does not increase absorption in as meaningful a manner as maintaining the original application. The second reason is the increased application time, along with the tighter sealing method, reduces evaporation of water resulting in a more moisturized and thus softer nail plate, with reduced tensile strength that is removed more easily. The porous nail plate with the diminished hydrophobic effect permits the urea and bifonazole to be more readily absorbed, thus increasing treatment efficacy. Application of urea over time loosens the nail keratin and improves nail hydration, which ultimately softens the nail and loosens the firm attachment of the nail plate to the underlying nail bed, thus aiding in easy removal of the infected portion of the nail.

Another finding that emerges from the results is that an increase in age of 1 year is associated with an increase in the time to chemical avulsion by 0.128 days. We also found that patients aged 60 and over had longer treatment duration to complete chemical avulsion on average in each of the treatment groups (group $1=19.42$ days; group $2=18.83$; group $3=12.85$ days). These findings can be explained by the thickening of the toenails with aging, which may make it more difficult to remove them using the ointment, difficulty bending and reaching the feet, and difficulty cutting (and scraping) the thick toenails, known phenomena among the elderly [16].

Due to these promising results, it is suggested that onychomycosis patients treated with the urea $40 \%$ and bifonazole $1 \%$ ointment apply the treatment to the affected nail once a week for 1 week and cover the nail using the new tight sealing method. This new treatment protocol is more efficacious and adequately tolerable (the only side effect which was reported by 2 patients in the once a week application treatment group was mild dermal irritation which did not prevent them from completing the treatment) and may improve patient compliance, as it requires less work from the patient with faster results. This is especially true for patients with onychomycosis of the toenails who have difficulty bending, such as older or physically disabled patients, or those who require assistance from caregivers. Although we did not find differences in treatment compliance between treatment groups, there may have been such that we were unable to detect due to follow-up that was not strict enough for precise adherence to treatment protocol.

The purpose of the treatment with the urea $40 \%$ and bifonazole $1 \%$ ointment is achieving mycological cure. 
Unfortunately, we did not have the information regarding this parameter as our research is retrospective, and this data was not collected. We suggest that future studies utilize our new treatment protocol to examine this parameter, in addition to the time to chemical avulsion, in order to demonstrate that our new treatment protocol achieves quicker chemical avulsion with similar mycological cure rates. Another parameter that was not available to us and could have contributed to the results was the thickness of initial toenails, as it could be included in the multiple regression analysis to investigate if once weekly application has the same efficacy in achieving chemical avulsion of toenails of increased thickness. We suggest conducting a randomized control trial in the near future that will examine the results of our study and include the parameters described above (mycological cure and toenail thickness).

\section{Statement of Ethics}

We conducted the research in accordance with the World Medical Association Declaration of Helsinki. We had Helsinki approval which was given to us by the Israeli Ministry of Health to conduct this retrospective cohort study in the "LEV HAYASMIN" clinic.

\section{Conflict of Interest Statement}

A.K. Gupta is a coordinating investigator for Moberg Pharma and Bausch Health Canada and a speaker for Bausch Health Canada. The authors have no other relevant affiliations or financial involvement with any organization or entity with a financial interest in or financial conflict with the subject matter or materials discussed in the manuscript apart from those disclosed. Other authors have no conflicts of interest to declare.

\section{Funding Sources}

The authors did not receive any funding. Mediprobe Research Inc. is a not-for-profit, self-funded research organization.

\section{Author Contributions}

Avner Shemer is the primary investigator and Yossi Eshel is the primary researcher in this project. Aditya K. Gupta contributed to the conceptualization and writing of the manuscript. Renata Farhi assisted in data collection from patient files. Eckart Haneke and Ralph Daniel also helped in conceptualization. Kelly Foley contributed to the statistical analysis and interpretation of the data. Emma Quinlan contributed to the writing of the manuscript.

\section{References}

1 Scher RK. Onychomycosis is more than a cosmetic problem. Br J Dermatol. 1994 Apr; 130(Suppl 43):15.

2 Gupta AK, Versteeg SG, Shear NH. Onychomycosis in the 21st century: an update on diagnosis, epidemiology, and treatment. $\mathrm{J} \mathrm{Cu}$ tan Med Surg. 2017 Dec;21(6):525-39.

3 Gupta A, Mays R. The impact of onychomycosis on quality of life: a systematic review of the available literature. Skin Appendage Disord. 2018;4: 208-16.

4 Beuscher T, Kelechi T. Onychomycosis: diagnosis, treatment, and prevention. J Wound Ostomy Cont Nurs. 2019 Aug;46(4): 333-5

5 Kaul S, Yadav S, Dogra S. Treatment of dermatophytosis in elderly, children, and pregnant women. Indian Dermatol Online J. 2017 Oct;8(5):310-8.

6 McAuley WJ, Jones SA, Traynor MJ, Guesné S, Murdan S, Brown MB. An investigation of how fungal infection influences drug penetration through onychomycosis patient's nail plates. Eur J Pharm Biopharm. 2016 May;102:178-84.
7 Elsayed MMA. Development of topical therapeutics for management of onychomycosis and other nail disorders: a pharmaceutical perspective. J Control Release. 2015 Feb;199: $132-44$.

8 Baraldi A, Jones SA, Guesné S, Traynor MJ, McAuley WJ, Brown MB, et al. Human nail plate modifications induced by onychomycosis: implications for topical therapy. Pharm Res. 2015 May;32(5):1626-33.

9 Seebacher C, Brasch J, Abeck D, Cornely O, Effendy I, Ginter-Hanselmayer G, et al. Onychomycosis. Mycoses. 2007 Jul;50(4):321-7.

10 Hay RJ. The future of onychomycosis therapy may involve a combination of approaches. $\mathrm{Br}$ J Dermatol. 2001 Oct;145(Suppl 60):3-8.

11 Zou Q, Habermann-Rottinghaus SM, Murphy KP. Urea effects on protein stability: hydrogen bonding and the hydrophobic effect. Proteins. 1998;31(2):107-15.
12 Jacobi A, Mayer A, Augustin M. Keratolytics and emollients and their role in the therapy of psoriasis: a systematic review. Dermatol Ther. 2015 Mar;5(1):1-18.

13 Wang B, Yang W, McKittrick J, Meyers MA. Keratin: structure, mechanical properties, occurrence in biological organisms, and efforts at bioinspiration. Prog Mater Sci. 2016 Mar 1; 76:229-318.

14 Ministry of Health, Israel. AGISPOR ONYCHOSET Prescribing Information [Internet]. 2014 [cited 2020 Mar 26]. Available from: https: //www.old.health.gov.il/units/pharmacy/ trufot/alonim/Agispor_dr_1410796308211. pdf.

15 Friedman-Birnbaum R, Cohen A, Shemer A, Bitterman O, Bergman R, Stettendorf S. Treatment of onychomycosis: a randomized, double-blind comparison study with topical bifonazole-urea ointment alone and in combination with short-duration oral griseofulvin. Int J Dermatol. 1997 Jan;36(1):67-9.

16 Baran R. The nail in the elderly. Clin Dermatol. 2011;29(1):54-60. 